\title{
AdS/CFT Correspondence and Type 0 String Theory*
}

\author{
Dario Martelli \\ SISSA, Via Beirut 2-4 Trieste 34014 \\ INFN Sezione di Trieste, Italy \\ dmartell@sissa.it
}

Abstract: We review some applications of Type 0 string theory in the context of the AdS/CFT correspondence.

Most of the success of the AdS/CFT correspondence is so far devoted to those situations where at least some fraction of supersymmetry is preserved. The case of Type IIB supergravity in $\mathrm{AdS}_{5} \times \mathrm{S}^{5}$ is a typical example [i], where a detailed mapping between quantities living in the two sides of the correspondence can be exploited. However, an important issue to address is how to embed the physically relevant nonsupersymmetric gauge theories in the correspondence, eventually recovering asymptotic freedom and confinement.

There are different proposals for giving a holographic description of nonsupersymmetric gauge theories, which basically deal with possible mechanisms for breaking supersymmetry. After recalling some of them, we will discuss some consequences of the approach proposed by Polyakov in [n-1 1 that consists in considering a nonsupersymmetric string theory with non-chiral GSO projec-

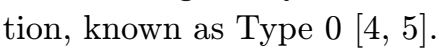

Before going to the main subject, we will briefly recall some of the alternative approaches presented in the literature.

It was pointed out by Witten $\left[6_{1}^{\prime \prime}, \overline{1}_{1}^{\prime}\right]$ that the AdS/CFT correspondence can be formulated at finite temperature. In doing so one identifies the Hawking temperature $T_{H}$ of the supergravity solution with that of the field theory in a thermal bath. The antiperiodic boundary conditions for fermions along the compactified (time) direction break supersymmetry and give them masses

\footnotetext{
*Preprint SISSA 17/2000/EP

${ }^{1}$ See [3] for recent developments.
}

$m_{f} \sim T_{H}$ at tree level. This leads also spin 0 bosons to acquire nonzero masses at one loop and spoils conformal symmetry because cancellations in the $\beta$-function do not occur any more. At large distances $\left(L \gg R_{c o m p} \sim \frac{1}{T_{H}}\right)$ the infrared effective theory is then expected to be pure YM in one lower dimension.

This approach captures some of the expected qualitative features of the quantum field theory, as a confining behavior of the Wilson loop (basically due to the presence of a horizon in the metric) and a mass-gap in the glue-ball spectrum. Moreover it provides a Lorentz invariant regularization scheme, as opposed for instance to the lattice regularization. Nonetheless it has some drawbacks: There is still coupling with the physics in higher dimension, as both the masses of glue-balls and of fermions are of the same order of the Hawking temperature. In addition, the $p$-dimensional 't Hooft coupling obeys

$$
\lambda_{p} \sim \lambda_{p+1} T_{H}
$$

where $T_{H}$ acts as UV cutoff. As the cutoff is removed $\left(T_{H} \rightarrow \infty\right), \lambda_{p+1}$ should approach zero, but this is opposite to the regime in which the supergravity approximation applies ${ }^{2}$.

Among various means to break supersymmetry there are the deformations of supersymmetric solutions. One searches for (domain wall) solutions interpolating between some AdS vacuum with $\mathcal{N}$ supersymmetries and a different vacuum

\footnotetext{
${ }^{2}$ In this respect the situation is similar to the lattice approach, where computations are possible at strong coupling.
} 
that can be AdS or not, and has $\mathcal{N}^{\prime}<\mathcal{N}{ }^{3}$ In the field theory side this is interpreted as turning on some relevant operators that drive the initial theory to an effective IR theory via RG flow of the couplings. Later we will mention an application of this construction in the context of Type 0 theory.

Another way to break supersymmetry is that of considering orbifolds theories, or branes at singularities. These two methods have been pursued extensively in the literature and here we will not comment them further on.

\section{Type 0 Strings and Gravity}

In this section we summarize some basic features of Type 0 string theories [4] $\left[\begin{array}{c}1 \\ 1\end{array}\right]$ and their gravity effective actions [īin]

Type 0 string theories are purely bosonic strings with modular invariant partition function and four sectors whose low laying fields are here summarized $^{4}$

\begin{tabular}{c|cc} 
& $(N S+, N S+)$ & $(N S-, N S-)$ \\
\hline 0A/0B & $\Phi, B_{\mu \nu}, g_{\mu \nu}$ & $T$ \\
& $(R+, R \mp)$ & $(R-, R \pm)$ \\
\hline 0A & $A_{\mu}^{(1)} A_{\mu \nu \rho}^{(1)}$ & $A_{\mu}^{(2)} A_{\mu \nu \rho}^{(2)}$ \\
0B & $A^{(1)} A_{\mu \nu}^{(1)} A_{\mu \nu \rho \sigma}^{(+)}$ & $A^{(2)} A_{\mu \nu}^{(2)} A_{\mu \nu \rho \sigma}^{(-)}$
\end{tabular}

There is a corresponding doubled set of D-branes coupling to RR-fields.

As pointed out in [i2] there are no open string tachyons on the world-volume of these branes, while there is a perturbative closed string tachyon that renders the Minkowski vacuum unstable. Nevertheless one should regard this as an indication that ten dimensional flat background is not stable, while there should exist other vacua in which the theory makes sense. AdS space seems in this respect a good candidate in that it allows for tachyonic modes.

$\mathcal{N}=(1,1)$ supersymmetry on the worldsheet makes these theories similar in some respect to supersymmetric Type II. For example

\footnotetext{
${ }^{3}$ See for instance the talks related to $\left[{ }_{8}\right]$ in this conference.

${ }^{4}$ The two entries in parenthesis refer respectively to left and right movers and the signs correspond to the choice of GSO projection. The upper (lower) signs in the RR sectors define Type 0A (0B) theory.
}

all tree level correlators of vertex operators of $(\mathrm{NS}+, \mathrm{NS}+)$ and $(\mathrm{R}+, \mathrm{R}+)$ fields are the same as Type II. Using these and other properties it is possible to derive an expression for the effective gravity action [īi] that, split in the NSNS and RR contributions, reads:

$$
\begin{array}{r}
S_{\mathrm{NSNS}}=\int \mathrm{d}^{10} x \sqrt{-g} \mathrm{e}^{-2 \Phi}(R \\
\left.-\frac{1}{12}|\mathrm{~d} B|^{2}+4|\mathrm{~d} \Phi|^{2}-\frac{1}{2}|\mathrm{~d} T|^{2}-V(T)\right) \\
S_{\mathrm{RR}}=\int \mathrm{d}^{10} x \sqrt{-g}\left(f(T)\left|F_{p+2}\right|^{2}+\cdots\right)
\end{array}
$$

The main novelties are coming from the tachyon couplings. In particular, there is a potential $V(T)$ which is an even function of the tachyon field, as well as functions $f(T)$ multiplying RR terms, that can be worked out perturbatively.

\section{A couple of examples}

Here we recall the basic ideas behind applications of the Type 0 construction, with the help of two of the earlier examples provided by Klebanov and Tseytlin in the papers [1 10 , 1 1 1, As in the usual Type II case, the idea is to consider a setup of D-branes of the theory, work out the gauge theory living on their world-volume, and eventually find the corresponding solution of the gravity theory in order to make comparisons and, hopefully, predictions.

\subsection{YM theory in 4 dimensions}

Consider the stack of $N$ D3 branes of the same type in Type 0B string theory. The configuration is stable and the low energy spectrum on their world-volume consists of $S U(N)$ gauge bosons plus 6 adjoint scalars in $3+1$ dimensions.

In $\left[100_{1}^{\prime}\right]$ the authors find two approximate solutions of the gravity equations of motions that involve nontrivial tachyon, dilaton, together with RR 4-form, and whose asymptotics are given in the UV and IR regions ${ }^{5}$.

The metric approaches in both cases $\mathrm{AdS}_{5} \times \mathrm{S}^{5}$ (with different radii) and the behavior of the other

\footnotetext{
${ }^{5}$ The identifications of these regimes follows from the usual identification of the radial coordinate transversal to the stack, with energy scale of the gauge theory.
} 
fields is as follows. The first one displays a vanishing 't Hooft coupling and a tachyon condensate with $\langle T\rangle \sim-1$, while the second represents an IR conformal point at infinite coupling — in fact the dilaton blows up while the tachyon condensate is $\langle T>\sim 0$.

Interestingly, the asymptotic freedom is reproduced. It should be noted however that in this situation $\alpha^{\prime}$ corrections become important, while in the IR one has to worry about string loop corrections. Moreover, connecting the two asymptotic solutions is still an open question.

\subsection{Nonsupersymmetric CFT}

Another interesting problem that has been studied is the construction of gauge theories that are conformal though nonsupersymmetric and their dual gravitational description. Let us illustrate the construction in [ ${ }_{1}^{1} i_{1}^{\prime}$. In Type 0B theory one starts with a stack of $N$ D3 branes of one type (say, electric) and $N$ of the other (say, magnetic). This configuration is argued to be stable in the large $N$ limit $^{6}$. The theory living on the worldvolume of this stack is a $S U(N) \times S U(N)$ gauge theory in $3+1$ dimensions, whose field content comprises, in addition to gauge bosons, 6 adjoint scalars for each $S U(N)$ factor, 4 bifundamental fermions in the $(\mathbf{N}, \overline{\mathbf{N}})$ and their conjugates. The dual gravity solution found in [1 $\left.{ }_{1}^{1}\right]$ is again $\mathrm{AdS}_{5} \times \mathrm{S}^{5}$ with constant dilaton and vanishing tachyon.

String loop suppression requires large $N$ as usual, while tachyon stability translates in a condition on the 't Hooft coupling, namely

$\lambda=g_{Y M}^{2} N \lesssim 100$. Then the dual theory should be a CFT in the large $N$ limit, for not very large coupling.

The gravity solution resembles very much the Type IIB familiar case. In fact, it was pointed out in $[12 \pi]$ that the gauge theory belongs to the class of orbifold theories of $\mathcal{N}=4 \mathrm{SYM}$, where the $Z_{2}$ projection belongs to the center of the $R$ symmetry group. So it is exactly conformal in the large $N$ limit. This information can be used to reverse the argument, that is, via $\mathrm{AdS} / \mathrm{CFT}$ the gauge theory may be predictive on the string

\footnotetext{
${ }^{6}$ At finite $\mathrm{N}$ branes of different types repel each other because of fermionic degrees of freedom in their worldvolume.
}

theory (or gravity) side. In fact the stability of the CFT at weak coupling implies that Type 0B theory on $\mathrm{AdS}_{5} \times \mathrm{S}^{5}$ should be stable for sufficiently small radius. In $\left[1 \overline{3}_{1}\right]$ it is suggested that the instability of the background at large radius translates in a phase transition occurring in the large $N$ CFT at strong coupling, with anomalous dimension of the operator dual to the tachyon field developing a singularity at a critical value $\lambda_{c}$.

\section{Non-critical Type 0}

In this section we expose some results obtained

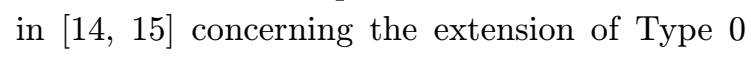
string theory in non-critical dimensions, together with a proposal for the effective action and the discussion of some solutions.

The original proposal in [2] was to consider a string theory in dimension $d<10$. Even if a microscopic description of string theory out of criticality is still far, there are at least indications for a possible extension of Type 0 theories in noncritical dimensions:

- a diagonal partition function, whose modular invariance doesn't rely on $d=10$ (as opposed to Type II theories)

- the tachyon should condense, providing an effective central charge $c_{\text {eff }} \sim V(\langle T\rangle)$. It doesn't seem unnatural to shift $c_{\text {eff }}$ by the central charge deficit $(10-d)$

$c_{\text {eff }}$ provides a tree level cosmological constant in the low energy theory, and this agrees with the expectation that the inconsistencies possibly arise only in flat background.

We work at the level of the effective gravity action. In $d<10$ one should guess the field content of the theory and write down the relative action. In [1 1 - 5 ] we assume the NSNS sector (gravity + tachyon) is always present and the RR sector is worked out on group theory grounds, considering tensor products of $S O(d-2)$ spinors. In five dimensions for instance, $\mathbf{2} \times \mathbf{2}=\mathbf{1}+\mathbf{3}$, and one is led to include a scalar potential $A$ and a vector $A_{\mu}$. We further assume the existence of a 4 -form potential to accommodate the would be 
D3-brane: This really amounts to consider massive gravity.

With these assumptions, the equations of motion following from the relative action have interesting solutions, whose interpretation may give some insight into their field theory duals, and eventually provide hints in favor of the consistency of either critical or even non-critical Type 0 string theory.

The relevant piece of the $d$-dimensional action, for the ansatz that we consider is ${ }^{7}$

$$
\begin{array}{r}
S=\int \mathrm{d}^{d} x \sqrt{-g}\{R \\
-\frac{1}{2}\left(\partial_{M} \Phi\right)^{2}-\frac{1}{2}\left(\partial_{M} T\right)^{2}-V(T) \mathrm{e}^{\sqrt{\frac{2}{d-2}} \Phi} \\
\left.-\frac{f(T)}{2(p+2) !} \mathrm{e}^{\frac{1}{2} \sqrt{\frac{2}{d-2}}(d-2 p-4) \Phi}\left(F_{M_{1} \cdots M_{p+2}}\right)^{2}\right\},
\end{array}
$$

where $V(T)=-10+d-\frac{d-2}{8} T^{2}+\cdots$ is the tachyon potential, including central charge deficit.

One wants to find solutions of the equations of motion following from the action above and give them a dual interpretation. Consider an ansatz in which nonzero fields are the metric, constant dilaton $\left(\Phi_{0}\right)$ and tachyon $\left(T_{0}\right)$, and a $\mathrm{RR}(p+2)$-form field strength:

$$
\begin{array}{r}
R_{\mu \nu \rho \lambda}=-\frac{1}{R_{0}^{2}}\left(g_{\mu \rho} g_{\nu \lambda}-g_{\mu \lambda} g_{\nu \rho}\right) \\
R_{i j k l}=+\frac{1}{L_{0}^{2}}\left(g_{i k} g_{j l}-g_{i l} g_{j k}\right) \\
F_{\mu_{1} \cdots \mu_{p+2}}=F_{0} \sqrt{-g_{(p+2)}} \epsilon_{\mu_{1} \cdots \mu_{p+2}} .
\end{array}
$$

Then the equations of motion become a set of algebraic equations. The tachyon VEV is determined implicitly by the following equation

$$
\frac{f^{\prime}\left(T_{0}\right)}{f\left(T_{0}\right)}=\frac{1}{2}(d-2 p-4) \frac{V^{\prime}\left(T_{0}\right)}{V\left(T_{0}\right)} .
$$

Now, without a precise knowledge of the functions $f(T)$ and $V(T)$ one cannot infer whether it admits solutions. One should really assume it has, and extract some information. The remaining equations fix the value of the radii of the two maximally symmetric spaces and that of

\footnotetext{
${ }^{7}$ Uppercase indices run from 1 to $d$, while Greek and Latin indices run from 1 to $p+2$ and from $p+3$ to $d$ respectively.
}

the dilaton. It turns out that such solution is a $\mathrm{AdS}_{p+2} \times \mathrm{S}^{d-p-2}$ space, with tachyon VEV, and fixed 't Hooft coupling $\lambda=e^{\Phi_{0}} N .{ }^{8}$

Notice that string loop corrections are suppressed in the large $N$ limit, while $\alpha^{\prime}$-corrections are important because the curvature is $O(1)$.

By the AdS/CFT correspondence the field theory dual of this solution should be at a conformal point. However it is difficult to make contact with perturbative field theory because $\lambda \sim O(1)$. Nevertheless one can still get additional information. It is in fact possible to count the number of degrees of freedom that should live in the theory dual to this background [1]

Consider a thermal deformation of the solution. Identifying the Hawking temperature with the finite temperature of the field theory one can compute the entropy. By either computing the free energy from the Euclidean action, or computing the area of the horizon, it turns out that it has the following behavior

$$
S \sim N^{2} V_{p} T_{H}^{p}
$$

for any value of $p$. This is an indication that the dual field theory should have $N^{2}$ degrees of freedom, and could be YM theory in some non Gaussian limit. Notice the different scaling power in the analogous relation one gets from evaluating the entropies of black M2 and M5 branes $(3 / 2$ and 3 respectively).

Notice that this kind of approach is potentially predictive. Consider in fact the formula for scaling dimensions of dual operators ${ }^{9}$

$$
\begin{gathered}
\Delta=\frac{(d-1)+\sqrt{(d-1)+4 m^{2} R_{0}^{2}}}{2} \\
m^{2} R_{0}^{2}=d(d-1)\left(1+\frac{\tau}{2} \pm \frac{1}{2} \sqrt{\tau^{2}+(2 d-4) \frac{V^{\prime}\left(T_{0}\right)^{2}}{V\left(T_{0}\right)^{2}}}\right) \\
\text { with } \quad \tau=d \frac{V^{\prime}\left(T_{0}\right)^{2}}{V\left(T_{0}\right)^{2}}-\frac{2}{d} \frac{f^{\prime \prime}\left(T_{0}\right)}{f\left(T_{0}\right)}-\frac{V^{\prime \prime}\left(T_{0}\right)}{V\left(T_{0}\right)}-1 .
\end{gathered}
$$

First, note that the tachyon VEV $T_{0}$ behaves as a "bare" quantity: it does not enter in determining physical quantities. It is very much as in

\footnotetext{
${ }^{8} N$ is the number of branes, which has to be evaluated in the string frame.

${ }^{9}$ These results are obtained in the case $d=p+2$.
} 
the renormalization group. One can do a field redefinition, this will shift $T_{0}$, without affecting $\lambda, R_{0}, \Delta$.

Then, the scaling dimensions depend on a $f$ nite number of parameters and with a good guess on the field theory side, one in principle should be able to predict an infinite tower of dimensions from KK analysis.

\section{Holographic RG Flows}

The extension of the AdS/CFT correspondence to theories away from their conformal limit leads naturally to the issue of giving a dual description of RG flows. This holographic description has recently attracted much attention. Here we will focus on a Type 0 application.

The construction of gravity solutions describing RG flows is quite general. Given 1) an effective potential for the scalars and 2) the existence of AdS solution (at extrema of the potential), the procedure is in principle straightforward. In fact the equations of motion for the coupled scalars plus gravity system provide the RG equations for the couplings in the dual theory, once an appropriate ansatz for the metric is plugged in as a physical input. This setup can be achieved considering consistent truncations of Type IIB or 11d supergravities (for instance gauged supergravities) or other KK compactifications emerging for example as compactifications on manifolds which are near horizon limit of branes at conical singularities. Finally, such a framework can be provided by a simple gravity theory, such as the Type 0 gravity.

In this case the effective action is:

$$
S=\int \mathrm{d}^{d} x \sqrt{-g}\left\{R-\frac{1}{2}\left(\partial \phi_{i}\right)^{2}-\mathcal{V}\left(\phi_{i}\right)\right\} .
$$

where $\phi_{i}=(\Phi, T)$. With the following ansatz

$$
\begin{array}{r}
\mathrm{d} s^{2}=\mathrm{d} y^{2}+A^{2}(y) \mathrm{d} \vec{x}^{2} \\
\phi_{i}=\phi_{i}(y)
\end{array}
$$

the equations of motions read

$$
\begin{array}{r}
\ddot{\phi}_{i}+\gamma \dot{\phi}_{i}=\partial_{i} \mathcal{V} \\
\frac{\ddot{A}}{A}+(d-2)\left(\frac{\dot{A}}{A}\right)^{2}=\frac{1}{d-2} \mathcal{V}
\end{array}
$$

$$
\dot{\gamma}=-\frac{d-1}{2(d-2)}\left(\dot{\phi}_{i}\right)^{2}
$$

where $\gamma=(d-1) \frac{\mathrm{d}}{\mathrm{d} y} \log A$.

Now, suppose that (3.2) has more than one solution, say $T_{1}, T_{2}$ at least. This means that exist two AdS solutions, with fixed values for scalars, and radii $R_{2}>R_{1}$, say. Then, it can be shown (see [is for instance) that there are interpolating solutions between the previous two. The explanation of this fact is roughly as follows. The set of equations (1. $\left(\begin{array}{l}3 \\ -1\end{array}\right)$ can be interpreted as describing a point-like particle rolling in a potential $-\mathcal{V}$, subject to an effective friction force encoded in $\gamma$. In fact, given that $\gamma$ is monotonically decreasing and

$$
\gamma \rightarrow \frac{d-1}{R_{2}}>0
$$

as $y \rightarrow \infty$, it follows that it is strictly positive along the trajectory. The solutions we are looking for are then those starting at a minimum of $\mathcal{V}$ for $y \rightarrow-\infty$ (IR) and ending at maximum for $y \rightarrow \infty$ (UV).

Some universal information can be read from the local behavior of these solutions. Consider for instance the UV fixed point. First it can be identified a coordinate which in the field theory can be consistently interpreted as the energy scale and parameterizes the interpolating solution. Such a coordinate can be chosen to be $\mathrm{e}^{10}$

$$
U=\frac{A^{2}}{\dot{A}} .
$$

The linearized equation for the fluctuations eigenvectors $\delta \tilde{\phi}_{i}(U)$ has the solutions

$$
\delta \tilde{\phi}_{i}=A_{i} U^{-\left(d-1-\Delta_{i}\right)}+B_{i} U^{-\Delta_{i}}
$$

where $\Delta_{i}$ were given in the previous section. One can then extract the leading $\beta$-functions for the running couplings ${ }^{11}$ :

$$
\beta_{i}\left(g_{i}\right)=U \frac{\mathrm{d}}{\mathrm{d} U} g_{i}=\left(\Delta_{i}-(d-1)\right)\left(g_{i}-g_{i} *\right)+\cdots
$$

\footnotetext{
${ }^{10}$ Different definitions can be given, which reduce locally to the same one.

${ }^{11}$ Unless $\Delta_{i}=d-1$, which means that there is a VEV for some operator in the dual field theory [8닌.].
} 


\section{Résumé and Perspectives}

We have illustrated how the Type 0 approach can be used to apply the AdS/CFT correspondence to nonsupersymmetric cases. This method offers some advantages compared to others approaches, as the finite temperature one for instance. Though, it has some limitations as well. We conclude summarizing some problems

- $\mathcal{N}=0 \rightarrow$ difficult to check $^{12}$

- there is no proof of tachyon condensation

- $\alpha^{\prime}$ corrections are usually important

- lack of a microscopic stringy description in $d<10$

and some interesting features

- cutoff independent results

- confining and asymptotically free solutions

- no mixing with higher dimensional physics

- "conformal" solutions

In particular the last point is potentially interesting. These solutions could be just an artifact of the approximation. However they can also indicate some novel field theories or (strongly coupled) conformal fixed points in YM theories as suggested in [2.

We conclude noticing that, some control on the field theory may shed light on Type 0 string theory and/or on non-critical string theory via AdS/CFT correspondence. In particular the latest developments in the context of holographic RG flows could provide an appropriate framework for studying which constraints the dual field imposes on the gravity theory.

\section{Acknowledgments}

I wish to thank the organizers of the conference for hospitality. This work was supported in part by European Union TMR program CT960045.

\footnotetext{
${ }^{12}$ This is common to all nonsupersymmetric approaches.
}

\section{References}

[1] J. Maldacena, "The large $\mathrm{N}$ limit of superconformal field theories and supergravity," Adv. Theor. Math. Phys. 2 (1998) 231 [hepth/9711200].

[2] A.M. Polyakov, "The wall of the cave," Int. J. Mod. Phys. A14 (1999) 645 [hep-th/9809057].

[3] A. Polyakov and V. Rychkov, "Gauge fields: Strings duality and the loop equation," [hepth/0002106].

[4] L.J. Dixon and J.A. Harvey, "String Theories In Ten-Dimensions Without Space-Time Supersymmetry," Nucl. Phys. B274, 93 (1986).

[5] N. Seiberg and E. Witten, "Spin Structures In String Theory," Nucl. Phys. B276, 272 (1986).

[6] E. Witten, "Anti-de Sitter space and holography," Adv. Theor. Math. Phys. 2 (1998) 253 [hep-th/9802150].

[7] E. Witten, "Anti-de Sitter space, thermal phase transition, and confinement in gauge theories," Adv. Theor. Math. Phys. 2 (1998) 505 [hepth/9803131].

[8] M. Petrini and A. Zaffaroni, "The holographic RG flow to conformal and non-conformal theory," [hep-th/0002172].

[9] I.R. Klebanov and A.A. Tseytlin, "D-branes and dual gauge theories in type 0 strings," Nucl. Phys. B546 (1999) 155 [hep-th/9811035].

[10] I. R. Klebanov and A. A. Tseytlin, "Asymptotic freedom and infrared behavior in the type 0 string approach to gauge theory," Nucl. Phys. B547 (1999) 143 [hep-th/9812089].

[11] I. R. Klebanov and A. A. Tseytlin, "A nonsupersymmetric large N CFT from type 0 string theory," JHEP 9903 (1999) 015 [hepth/9901101].

[12] N. Nekrasov and S. L. Shatashvili, "On NonSupersymmetric CFT in Four Dimensions," [hep-th/9902110].

[13] I. R. Klebanov, "Tachyon stabilization in the AdS/CFT correspondence," Phys. Lett. B466 (1999) 166 [hep-th/9906220].

[14] G. Ferretti and D. Martelli, "On the construction of gauge theories from non critical type 0 strings," Adv. Theor. Math. Phys. 3 (1999) 119 [hep-th/9811208].

[15] G. Ferretti, J. Kalkkinen and D. Martelli, "Noncritical type 0 string theories and their field theory duals," Nucl. Phys. B555 (1999) 135 [hepth/9904013]. 